PROCEEDINGS OF THE

AMERICAN MATHEMATICAL SOCIETY

Volume 134, Number 3, Pages 779-791

S 0002-9939(05)07998-0

Article electronically published on July 19, 2005

\title{
POISSON GEOMETRY OF DIFFERENTIAL INVARIANTS OF CURVES IN SOME NONSEMISIMPLE HOMOGENEOUS SPACES
}

\author{
G. MARÍ BEFFA
}

(Communicated by Jozef Dodziuk)

\begin{abstract}
In this paper we describe a family of compatible Poisson structures defined on the space of coframes (or differential invariants) of curves in flat homogeneous spaces of the form $\mathcal{M} \cong\left(G \ltimes \mathbb{R}^{n}\right) / G$ where $G \subset \operatorname{GL}(n, \mathbb{R})$ is semisimple. This includes Euclidean, affine, special affine, Lorentz, and symplectic geometries. We also give conditions on geometric evolutions of curves in the manifold $\mathcal{M}$ so that the induced evolution on their differential invariants is Hamiltonian with respect to our main Hamiltonian bracket.
\end{abstract}

\section{INTRODUCTION}

Most known completely integrable systems can be integrated with the use of two Hamiltonian structures. It is well known that, if the system is Hamiltonian with respect to two compatible Hamiltonian structures (that is, it is bi-Hamiltonian) and one structure is non-degenerate, a recursion operator can be found and with it a sequence of integrals of the flow. This family of preserved quantities integrates the system (see [Ma]).

The relationship between completely integrable systems and classical geometry of curves was fully established when Hasimoto $\mathrm{Ha}$ showed how the curvature and torsion of the vortex filament flow satisfied a nonlinear Schrödinger equation, a wellknown integrable system. Furthermore, it is was pointed out ([LP1], [LP2]) that one of the Hamiltonian structures used to integrate the NLS flow could be defined using the Euclidean geometry of the flow itself. This situation was also known to be true for a family of projective flows linked to KdV type equations (DS, M4). That is, if a flow is invariant under the action of the group $(G=O(n)$ in the Euclidean case and $G=\operatorname{PSL}(n, \mathbb{R})$ in the projective case) the question of integrability could be shifted to a question of integrability of the flow of the differential invariants (what we call the invariantization of the flow) and, with it, to the construction and study of compatible Hamiltonian structures in this space. Several integrable systems have been obtained this way by looking at the evolution of the differential invariants of curves in different geometries and by trying to find known completely integrable systems among them (see [SW], KQ1, KQ2 and the references within) although

Received by the editors August 20, 2004 and, in revised form, October 15, 2004.

2000 Mathematics Subject Classification. Primary 37K25; Secondary 37K05, 37K10, 53A55.

Key words and phrases. Invariant evolutions of curves, homogeneous spaces, infinite dimensional Poisson geometry, differential invariants, completely integrable PDEs.

(C)2005 American Mathematical Society Reverts to public domain 28 years from publication 
there was no study of the Hamiltonian structures themselves or of the relationship of the Hamiltonian structures to geometry.

In a series of recent papers, the author identified Hamiltonian structures defined by the geometry of curves in Euclidean and conformal flat spaces ( $\mathrm{M} 2$, M3]). The present paper generalizes the construction done in $\mathrm{M} 3$ to obtain a family of Hamiltonian structures associated to the geometry of curves in a flat homogeneous space of the form $\left(G \ltimes \mathbb{R}^{n}\right) / G$ with $G \subset \mathrm{GL}(n, \mathbb{R})$ semisimple. This includes Euclidean, affine, special affine, Lorentz and symplectic geometries among others. The condition of semisimplicity is fundamental to the construction of the bracket, but the requirement that the space is flat is included because of the lack of knowledge on what the precise role of the curvature in the production of Poisson brackets is. The basic idea is that one can find a foliation $\Phi$ in $\mathcal{L} \mathfrak{g}^{*}$ (the space of loops on the dual of the Lie algebra $\mathfrak{g}$ ) and an open set $U \subset \mathcal{L} \mathfrak{g}^{*}$ such that the quotient $U / \Phi$ is identified locally with the space of differential invariants of curves in $\left(G \ltimes \mathbb{R}^{n}\right) / G$ (Theorem 4). There are well-known compatible Poisson brackets defined on $\mathcal{L} \mathfrak{g}^{*}$, and we show that they can be Poisson reduced to the quotient $U / \Phi$, generating a family of compatible Poisson structures in the space of differential invariants (Theorems 5 and 6). Among the structures in this family, the one obtained when we reduce the so-called Kac-Moody Lie-Poisson structure is directly linked to flows of geometric evolutions in $\left(G \ltimes \mathbb{R}^{n}\right) / G$. In fact, it has been suggested that the invariantization of the Euler-Lagrange equation could be directly related to this special Poisson bracket. We give conditions on geometric evolutions in $\left(G \ltimes \mathbb{R}^{n}\right) / G$ ensuring that the corresponding invariantization is Hamiltonian with respect to the reduced Poisson bracket (Theorem 8). Theorem 1 also establishes the relation between classical and group based moving frames.

\section{Preliminaries}

2.1. Moving frames. The classical concept of a moving frame is that of an invariant curve in the frame bundle along a curve $u$ in the manifold. Cartan's original idea was that differentiating such a classical moving frame will produce enough differential invariants to determine the curve itself. In fact, if one thinks briefly about the Euclidean case one of the differential invariants (the arc-length) is needed in advance in order to produce the classical Euclidean moving frame and cannot be produced by it. This problem appears again in conformal geometry, where a classical frame can only generate $n-2$ of the $n$ generating differential invariants (see $\mathrm{F}$, the conformal arc-length is one of two invariants that the classical frame equation fails to produce). On the other hand, a different concept of moving frame, that of an equivariant map defined on the jet space of the manifold, produces a complete system of generating differential invariants, as was shown in [FO1] and [FO2].

Let $\mathcal{M}$ be a manifold and let $J^{(k)}(\mathcal{M})$ be the $k$ th order jet bundle or set of equivalence classes of curves under the equivalence relation of $k$ th order contact. If we introduce coordinates $u=\left(u^{\alpha}\right)$ on $\mathcal{M}, x$ is the parameter and we denote by $u_{r}^{\alpha}=\frac{d^{r} u^{\alpha}}{d x^{r}}$, we can introduce coordinates in $J^{(k)}(\mathcal{M})$ given by $\left(x, u^{(k)}\right)=$ $\left(x, u^{\alpha}, u_{1}^{\alpha}, u_{2}^{\alpha}, \ldots, u_{k}^{\alpha}\right)$.

Definition 1. If a transformation group $G$ acts on $\mathcal{M}$, the action preserves the order of contact between curves and so there is an induced action in $J^{(k)}(\mathcal{M})$ called 
the prolonged action or $k$ th prolongation. It is locally given by

$$
g \cdot\left(x, u^{(k)}\right)=g \cdot\left(x, u, u_{1}, \ldots, u_{k}\right)=\left(x, g \cdot u,(g \cdot u)_{1}, \ldots,(g \cdot u)_{k}\right) .
$$

A ( $k$ th order) differential invariant is a local scalar function $I: J^{(k)}(\mathcal{M}) \rightarrow \mathbb{R}$ invariant under the prolonged action of $G$, that is, such that $I\left(g \cdot\left(x, u^{(k)}\right)\right)=$ $I\left(x, u^{(k)}\right)$ for all $g \in G$.

A $k$ th order relative differential invariant with Jacobian weight is a vector function $V: J^{(k)}(\mathcal{M}) \rightarrow \mathbb{R}^{n}$ such that

$$
V\left(g \cdot\left(x, u^{(k)}\right)\right)=J_{g}(u) V\left(x, u^{(k)}\right)
$$

where $J_{g}$ is the Jacobian matrix of the map $\phi_{g}: \mathcal{M} \rightarrow \mathcal{M}$ given by $\phi_{g}(u)=$ $g \cdot u$. That is, $J_{g}(u)=d \phi_{g}(u)$ (see [O] for more details on the Jacobian multiplier representation).

A $k$ th order left (resp. right) moving frame is a smooth $G$-equivariant map

$$
\rho: J^{(k)}(\mathcal{M}) \rightarrow G
$$

where we consider the left (resp. right) action of $G$ on itself and the prolonged action of the group on $J^{(k)}(\mathcal{M})$.

A theorem by Ovsiannikov $\mathrm{Ov}$, corrected by Olver in $\mathrm{O} 2$, states that if a group acts locally effectively on subsets, then there exists an integer $k_{0}$ such that the prolonged action of the given group is locally free on an open and dense subset of the $k_{0}$ th order jet space. This subset is formed by the so-called regular jets. A theorem in [. states that a moving frame exists locally if and only if $G$ acts freely and regularly in a neighborhood of the point. Hence, one can always raise the order of the jet space enough to guarantee the existence of moving frames on a neighborhood of regular jets (regular jets correspond to what is usually called nondegenerate curves in a given geometry, i.e., curves for which differential invariants can be found). Fels and Olver (FO1, FO2 $)$ have recently developed a general method to generate explicitly moving frames, including the frames presented here. One of the most important features of their method is that right moving frames can be found by solving the so-called normalization equations, equations of the form

$$
g \cdot\left(u, u_{1}, \ldots, u_{k}\right)=\left(c_{0}, c_{1}, \ldots, c_{k}\right)
$$

for some constant vectors $c_{i} \in \mathbb{R}^{n}$. The discussion above guarantees that, if we raise the order $k$ high enough and for a proper choice of constants $c_{i}$, the equations will determine $g$ and the resulting element

$$
g=\rho\left(u^{(k)}\right)
$$

will be a right moving frame. A left moving frame (we will need left moving frames for our study) will be given by its inverse. See [FO1] and [FO2] for more details.

One of the many advantages of this kind of moving frames (I will refer to them as group based moving frames) versus the classical kind is as follows.

Definition 2. Suppose $G$ acts freely and regularly on an open set of $J^{(k)}(\mathcal{M})$. Let $\rho$ be a left (resp. right) moving frame and let $\xi=\omega\left(\rho_{x}\right)$ be the pull-back of the left (resp. right) invariant Maurer-Cartan form on $G$. Then $\xi$ is called the left (resp. right) moving coframe associated to $\rho$. 
The entries of a moving coframe provide a generating set of differential invariants although there could be algebraic relations among them (see [FO1], [FO2]). If $G$ is a matrix group, the moving coframe will be the element in the algebra defining the differential equation satisfied by $\rho$, that is, the group based version of the Frenet equations. But, in the group based version all differential invariants appear in the entries of the moving coframe, including, in the Euclidean case, the arc-length (see [FO2) and, in the conformal case, the two independent differential invariants missing in the classical frame equation in $\mathrm{F}$ ( see [M2]).

Classical frames and relative differential invariants with Jacobian weight are the same concept: the first one emphasizes geometric properties while the second emphasizes the invariance under the prolonged action (see [O]). The following theorem describes the relation between classical and group based moving frames. This relation was described by the author in M1 for flat semisimple homogeneous spaces.

Theorem 1. Assume $\mathcal{M} \cong G / H$ is a homogeneous space and let $o \in \mathcal{M}$ be the class of $H$. Let $u: I \rightarrow \mathcal{M}$ be a nondegenerate curve and let

$$
\rho: J^{(m)}(\mathcal{M}) \rightarrow G
$$

be a group based left moving frame such that $\rho\left(u^{(m)}\right) \cdot o=u$. Consider $d \phi_{\rho\left(u^{(m)}\right)}(o)$ (the Jacobian matrix of $\phi_{\rho\left(u^{(m)}\right)}$ at o) as an element in $\mathrm{GL}(n, \mathbb{R})$. Then, the matrix $d \phi_{\rho\left(u^{(m)}\right)}(o)$ contains in columns a classical moving frame. That is, $d \phi_{\rho\left(u^{(m)}\right)}(o)$ is a nondegenerate matrix of relative differential invariants with Jacobian weight.

Proof. The proof is very simple. Since $\rho$ is a left moving frame, for any $g \in G$,

$$
\rho\left(g \cdot u^{(m)}\right)=g \rho\left(u^{(m)}\right) .
$$

Now, since $\rho\left(u^{(m)}\right) \cdot o=u$ and $\rho\left(g \cdot u^{(m)}\right) \cdot o=g \cdot u$ for all $g \in G$, we obtain by differentiation

$$
d \phi_{\rho\left(g \cdot u^{(m)}\right)}(o)=d \phi_{g}(u) d \phi_{\rho\left(u^{(m)}\right)}(o)=J_{g} d \phi_{\rho\left(u^{(m)}\right)}(o) .
$$

It is trivial to see that each column of $d \phi_{\rho\left(g \cdot u^{(m)}\right)}(o)$ is simply the corresponding column in $d \phi_{\rho\left(u^{(m)}\right)}(o)$ with $u^{(m)}$ substituted by $g \cdot u^{(m)}$. Hence, each column in $d \phi_{\rho\left(u^{(m)}\right)}(o)$ is a relative differential invariant with Jacobian weight and so the theorem follows.

2.2. Poisson reduction. Let $G \subset \operatorname{GL}(n, \mathbb{R})$ be a semisimple Lie group, and $\mathfrak{g}$ its Lie algebra. Let $\mathcal{L} G=C^{\infty}\left(S^{1}, G\right)$ be the group of loops on $G$ and let $\mathcal{L} \mathfrak{g}=$ $C^{\infty}\left(S^{1}, \mathfrak{g}\right)$ be its Lie algebra. Let $\mathcal{L} \mathfrak{g}^{*}=C^{\infty}\left(S^{1}, \mathfrak{g}^{*}\right)$ be the regular part of the dual, dense in the dual of the algebra of loops. There exists a well-known Poisson bracket defined on this space. If $\mathcal{H}$ and $\mathcal{G}$ are two functionals defined on $\mathcal{L} \mathfrak{g}^{*}$ and $B$ is the Killing form of $\mathfrak{g}$, then their Poisson bracket is given by the formula

$$
\{\mathcal{H}, \mathcal{G}\}(M)=\int_{S^{1}} B\left(\frac{\delta \mathcal{G}}{\delta L},\left(\frac{\delta \mathcal{H}}{\delta L}\right)_{x}+a d^{*}\left(\frac{\delta \mathcal{H}}{\delta L}\right)(M)\right) d x,
$$

where $\frac{\delta \mathcal{H}}{\delta L}$ is the variational derivative of $\mathcal{H}$ at $M, M \in \mathcal{L} \mathfrak{g}^{*}$.

For us, the fundamental property of this Poisson bracket is the fact that its symplectic leaves (the leaves where the flow of Hamiltonian systems lie) coincide with the orbits in $\mathcal{L} \mathfrak{g}^{*}$ under the action of the group of loops $\mathcal{L} G$ given by

$$
A(g)(M)=g^{-1} g_{x}+A d^{*}(g) M
$$


whose infinitesimal action of the Lie algebra $\mathcal{L} \mathfrak{g}$ is given by

$$
a(N)(M)=N_{x}+a d^{*}(N)(M) .
$$

See [PS] for more information.

This action can also be viewed as the action on solutions of

$$
\frac{d X}{d x}=X M
$$

where $M \in \mathcal{L} \mathfrak{g}^{*}$ is identified with a matrix the usual way. Indeed, if $Y=X g$ with $g \in \mathcal{L} G$, then the equation (2.4) associated to $Y$ is $\frac{d Y}{d x}=Y A(g) M$. Notice that, if $M$ is periodic, a solution $X$ will have a monodromy; that is, there exists $\Gamma \in G$ such that $X(x+p)=\Gamma X(x)$ for all $x$, where $p$ is the period.

The bracket (2.1) is known to be compatible with the family of brackets

$$
\{\mathcal{H}, \mathcal{G}\}_{0}(M)=\int_{S^{1}} B\left(\frac{\delta G}{\delta L}, a d^{*}\left(\frac{\delta \mathcal{H}}{\delta L}\right)\left(H_{0}\right)\right) d x
$$

for any choice of constant element $H_{0} \in g^{*}$. We will now describe the Poisson reduction procedure. It was originally described in $[\mathrm{MR}]$. We have modified the statement to make it simpler since the general version is not needed here. Let $(\mathcal{P},\{\}$,$) be a Poisson manifold and let E \subset T \mathcal{P}$ be a subbundle of the tangent to $\mathcal{P}$. Assume the following conditions are satisfied: (a) $E \cap T \mathcal{P}$ is integrable and defines a foliation on $\mathcal{P}$. We will call the foliation $\Phi$. (b) The space of leaves is a manifold and $\pi: \mathcal{P} \rightarrow \mathcal{P} / \Phi$ is a submersion. (c) $E$ leaves $\{$, $\}$ invariant. That is, if $\mathcal{H}, \mathcal{G}: \mathcal{P} \rightarrow \mathbb{R}$ are smooth functions on $\mathcal{P}$ with differentials vanishing on $E$, then the differential of $\{\mathcal{H}, \mathcal{G}\}$ also vanishes on $E$. One says that $E$ preserves the Poisson bracket $\{$,$\} .$

Definition 3. We say $(\mathcal{P}, E,\{\}$,$) is Poisson reducible if \mathcal{P} / \Phi$ has a Poisson structure, $\{,\}_{R}$ such that for any smooth $h, g: \mathcal{P} / \Phi \rightarrow \mathbb{R}$ and for any smooth extensions $\mathcal{H}, \mathcal{G}$ of $h \circ \pi$ and $g \circ \pi$ with differentials vanishing on $E$ we have

$$
\{\mathcal{H}, \mathcal{G}\}=\{h, g\}_{R} \circ \pi .
$$

The following theorem can be found in $\mathrm{MR}$ for the finite-dimensional case, although it can be applied to infinite-dimensional cases, as the authors state.

Theorem 2 (Reduction Theorem). Assume (P $, E,\{\}$,$) satisfy assumptions (a)-$ (b)-(c). Then, $(\mathcal{P}, E,\{\}$,$) is Poisson reducible.$

\section{Poisson BRACKets on the SPACE of DifFEREnTial INVARIANTS}

In this section we will describe how the Poisson brackets (2.1) and (2.5) reduce to the space of moving coframes locally around a curve $u$. The idea is that, locally, any element in an open set $U$ of $\mathcal{L} \mathfrak{g}^{*}$ can be taken to a moving coframe via the action (2.2) where $g$ is in $\mathcal{L} N$, a certain subgroup of $\mathcal{L} G$. First of all we proceed to identify the key elements in the reduction.

Assume $G$ is a semisimple matrix group and assume the nonsemisimple group $G \ltimes \mathbb{R}^{n}$ acts on $\mathbb{R}^{n}$ as

$$
(g, v) \cdot u=g u+v
$$


The group $G \ltimes \mathbb{R}^{n}$ can be viewed as the subgroup of $\mathrm{GL}(n+1, \mathbb{R})$ given by elements of the form $\left(\begin{array}{ll}1 & 0 \\ v & g\end{array}\right)$ acting on $\mathbb{R}^{n}$ as

$$
\left(\begin{array}{ll}
1 & 0 \\
v & g
\end{array}\right)\left(\begin{array}{l}
1 \\
u
\end{array}\right)=\left(\begin{array}{c}
1 \\
g u+v
\end{array}\right)
$$

Hence, a moving frame along a curve $u: J \rightarrow \mathbb{R}^{n}$ would be of the form

$$
\rho=\left(\begin{array}{cc}
1 & 0 \\
\rho_{u} & \rho_{G}
\end{array}\right)
$$

and its associated moving (left) coframe will be given by

$$
\rho^{-1} \rho_{x}=\left(\begin{array}{cc}
0 & 0 \\
\rho_{G}^{-1}\left(\rho_{u}\right)_{x} & \rho_{G}^{-1}\left(\rho_{G}\right)_{x}
\end{array}\right) .
$$

Theorem 3. There exists a left moving frame $\rho$ such that $\rho_{u}=u$ and such that $\rho_{G}^{-1}\left(\rho_{u}\right)_{x}$ contains all first-order differential invariants.

Proof. The proof of this theorem is based on the application of Fels and Olver's normalization procedure. Instead of finding a left moving frame we will find first a right invariant one by solving Fels and Olver's normalization equations. The zero-order normalization equation is given by

$$
\hat{\rho} \cdot u=g u+v=0,
$$

which is solved by choosing $v=-g u$. The first-order normalization equation is given by

$$
\hat{\rho} \cdot u_{1}=g u_{1}=c_{1}
$$

for some nonzero constant $c_{1} \in \mathbb{R}^{n}$. In some cases (for example $G=O(n)$ or $G=O(n-1,1))$ this equation does not have maximal rank and so $g u_{1}$, after $g=\hat{\rho}\left(u^{(k)}\right)$ has been found, is not in general $c_{1}$ but rather the first-order differential invariants (see [FO1], [FO2]). As indicated in the preliminaries, completing the normalization procedure results in the right invariant moving frame

$$
\hat{\rho}=\left(\begin{array}{cc}
1 & 0 \\
-g u & g
\end{array}\right) .
$$

The left invariant one will be its inverse, namely

$$
\rho=\left(\begin{array}{cc}
1 & 0 \\
\rho_{u} & \rho_{G}
\end{array}\right)=\left(\begin{array}{cc}
1 & 0 \\
u & g^{-1}
\end{array}\right) .
$$

Clearly $\rho_{G}^{-1}\left(\rho_{u}\right)_{x}=g u_{1}=c_{1}$ and so the theorem is proved.

Example 1. Our running example in this paper will be $G=\operatorname{SL}(2, \mathbb{R})$ or the geometry of special affine transformations on the plane. In this case, the zero-order normalization equations

$$
\left(\begin{array}{ll}
1 & 0 \\
v & F
\end{array}\right)\left(\begin{array}{l}
1 \\
u
\end{array}\right)=\left(\begin{array}{l}
1 \\
0
\end{array}\right)
$$

determine $v=-F u$. If we denote $u_{k}=\frac{d^{k} u}{d x^{k}}$ and if $\left\{e_{1}, e_{2}\right\}$ is the usual basis of $\mathbb{R}^{2}$, then the first-order normalization equations

$$
\left(\begin{array}{ll}
1 & 0 \\
v & F
\end{array}\right)\left(\begin{array}{c}
0 \\
u_{1}
\end{array}\right)=\left(\begin{array}{c}
0 \\
e_{1}
\end{array}\right)
$$


produce $F^{-1} e_{1}=u_{1}$. The second-order normalization equations

$$
\left(\begin{array}{cc}
1 & 0 \\
v & F
\end{array}\right)\left(\begin{array}{c}
0 \\
u_{2}
\end{array}\right)=\left(\begin{array}{l}
0 \\
0 \\
*
\end{array}\right)
$$

and the condition $F \in \operatorname{SL}(2, \mathbb{R})$ determine $F^{-1} e_{2}=\frac{u_{2}}{d}$ where $d=\operatorname{det}\left(u_{1}, u_{2}\right)$ (* above denotes an equation we are not using to normalize). Therefore, the left moving frame is given by

$$
\rho=\left(\begin{array}{cc}
1 & 0 \\
-F^{-1} v & F^{-1}
\end{array}\right)=\left(\begin{array}{ccc}
1 & 0 & 0 \\
u & u_{1} & \frac{u_{2}}{d}
\end{array}\right) .
$$

The $\mathrm{SL}(2, \mathbb{R})$ block provides a standard moving frame along the curve, namely $\left\{u_{1}, \frac{u_{2}}{d}\right\}$. A nondegenerate curve is one for which $d \neq 0$ and we can assume that $d>0$. Finally, the moving coframe is given by

$$
\rho^{-1} \rho_{x}=\left(\begin{array}{ccc}
0 & 0 & 0 \\
1 & 0 & \frac{\operatorname{det}\left(u_{3}, u_{2}\right)}{\operatorname{det}\left(u_{1}, u_{2}\right)} \\
0 & \operatorname{det}\left(u_{1}, u_{2}\right) & 0
\end{array}\right) .
$$

Clearly $k_{1}=\operatorname{det}\left(u_{1}, u_{2}\right)=d$ and $k_{2}=\frac{\operatorname{det}\left(u_{3}, u_{2}\right)}{\operatorname{det}\left(u_{1}, u_{2}\right)}$ are two generating differential invariants of the action. The invariant $k_{1}$ is the affine arc-length.

Assume that the first-order differential invariants of the curve are all constant either because the prolonged group action is transitive on an open subset of $J^{1}$, so there are no first-order differential invariants (such is the case in our running example) or because we can make them somehow constant. In all known cases this can be achieved by choosing $x$ to be a special parameter of arc-length type (such is the case in Euclidean geometry where the order of the arc-length is one). Under these assumptions $\rho_{G}^{-1}\left(\rho_{u}\right)_{x}=\Lambda \in \mathbb{R}^{n}$. The subgroup we will use for our reduction is defined as $\mathcal{L} N$, where $N \subset G$ is the stabilizer of $\Lambda$, that is

$$
N=\{g \in G \text { such that } g \Lambda=\Lambda\} .
$$

Let $E$ be the distribution defined by the Hamiltonian vector fields $\xi_{\mathcal{H}}$, Hamiltonian with respect to the bracket (2.1), and such that $\frac{\delta \mathcal{H}}{\delta M}(M) \in \mathcal{L} \mathfrak{n}$, where $\mathfrak{n}$ is the Lie algebra of $N$. It is known that $E$ is integrable and its leaves are the $\mathcal{L} N$-orbits of the action (2.2).

Let $\mathcal{K}$ be the set of elements of the form $\rho_{G}^{-1}\left(\rho_{G}\right)_{x}$, where $\rho_{G}$ is defined by the moving frame (3.1) associated to curves locally in a neighborhood of $u$.

Theorem 4. There exists an open subset of $\mathcal{L}^{*}, U$, such that $U / E \cong \mathcal{K}$.

Proof. We will show that, in an open set of $\mathcal{L g}^{*}$, any point is taken to $\mathcal{K}$ by the action (2.2) of a unique element in $\mathcal{L} N$. For convenience we will identify $\mathfrak{g}$ with $\mathfrak{g}^{*}$ using the nondegenerate Killing form.

Let $m \in \mathcal{L}^{*}$ and let $\rho(x) \in G$ be such that $\rho^{-1} \rho_{x}=m$. To obtain $\rho$ we would need to extend $m$ to a left invariant vector field in the group and solve the associated equations. Once we have obtained $\rho$ we choose $u$ to be a nondegenerate curve (that is, a curve whose jet is a regular jet in $J^{(k)}(\mathcal{M})$ ) such that $\rho \Lambda=u_{1}$. Due to the continuous dependence of solutions of ODEs on parameters this condition will determine an open set in $\mathcal{L g}^{*}$, locally around $u$ since $u$ itself is nondegenerate. Let $\rho_{G}$ be the $G$ component (or classical moving frame) of the moving frame associated 
to $u$ as in (3.1). Let $g_{0}=\rho^{-1} \rho_{G}$. We simply need to show that $g_{0} \in \mathcal{L N}$ and the proof will be finished.

Indeed, since both $\rho$ and $\rho_{G}$ take $\Lambda$ to $u_{1}, g_{0}$ will belong to the stabilizer of $\Lambda$. Furthermore, $g_{0}$ is periodic since we will prove that both $\rho$ and $\rho_{G}$ have the same monodromy. Let $\Gamma \in G$ be such that $\rho(x+T)=\Gamma \rho(x)$ for any $x$. Applied to $\Lambda$ this implies $u_{1}(x+T)=\Gamma u_{1}(x)$ and the same property would hold for a good choice of $u$. Since $\rho_{G}$ is determined uniquely by a fixed choice of normalization constants, we clearly obtain $\rho_{G}(x+T)=\Gamma \rho_{G}(x)$.

We will next apply Theorem 2 to $U$ and $E$ to quickly obtain a Poisson bracket on $\mathcal{K}$. This bracket will be our main Poisson bracket among the family we will reduce. In fact, it can be directly linked to geometric evolutions of curves in $\mathcal{M}$, as we will see in the next section.

Theorem 5. If we choose $U=\mathcal{P}$ and $E$ as above, Theorem 2 can be applied and the bracket (2.1) is Poisson reducible to $\mathcal{K}$. The resulting bracket is defined by relation (2.6).

Proof. The application of Theorem 2 is very simple. Indeed, (a) and (b) are known to be true. So the only condition that remains to be proved is the preservation of the bracket by $E$. If $\mathcal{F}$ and $\mathcal{H}$ are two Hamiltonian functionals whose variational derivatives vanish on $E$, then it is immediate to check that their Hamiltonian vector fields $\xi_{\mathcal{F}}(M)$ and $\xi_{\mathcal{H}}(M)$ vanish on $\mathfrak{n}$ for any $M \in U$. Therefore, if a functional $\mathcal{R}$ is such that its variational derivative belongs to $\mathfrak{n}$, we have

$$
\{\mathcal{H}, \mathcal{R}\}(M)=\{\mathcal{F}, \mathcal{R}\}(M)=0
$$

for any $M \in U$. Application of Jacobi's identity guarantees (c).

Finally, we look at the brackets (2.5). They will trivially reduce to $U / E$, and hence to $\mathcal{K}$, for any choice of $H_{0} \in \mathfrak{g}$ and they will provide us with a family of Poisson brackets compatible with $\{,\}_{R}$.

Theorem 6. The Poisson brackets (2.5) are also Poisson reducible to $\mathcal{K}$ for any choice of $H_{0} \in \mathfrak{g}$.

Proof. Again, we only need to check that the bracket preserves the foliation. The fact that the brackets do not depend on the point $M$ makes the proof of this condition trivial.

Example 2. We will use here the definition of reduced Poisson bracket to calculate them in the case $G=\mathrm{SL}(2, \mathbb{R})$. Let $h, f: \mathcal{K} \rightarrow \mathbb{R}$ be two functionals defined on $\mathcal{K}$, where

$$
\mathcal{K}=\left\{\left(\begin{array}{cc}
0 & k_{2} \\
k_{1} & 0
\end{array}\right), k_{1}>0, k_{1}, k_{2} \in C^{\infty}\left(S^{1}\right)\right\} .
$$

Let $\mathcal{H}$ and $\mathcal{F}$ be two extensions of $h$ and $f$ to $\mathcal{L} \operatorname{sl}(2, \mathbb{R})^{*}$ constant on the leaves of $E$. That is, if $H=\frac{\delta \mathcal{H}}{\delta M}(K)$ and $F=\frac{\delta \mathcal{F}}{\delta M}(K)$ are their variational derivatives at $K \in \mathcal{K}$, then

$$
H_{x}+[K, H] \in \mathfrak{n}^{0}, \quad F_{x}+[K, F] \in \mathfrak{n}^{0}
$$

where $\mathfrak{n}^{0}$ is the annihilator in $\mathcal{L} \operatorname{sl}(2, \mathbb{R})^{*}$ of the Lie subalgebra $\mathfrak{n}$ associated to the subgroup $N$ defined in (3.3). In our case $\Lambda=e_{1}$ and $\mathfrak{n}^{0}$ is formed by matrices of 
the form

$$
\left(\begin{array}{cc}
\alpha & \beta \\
0 & -\alpha
\end{array}\right)
$$

Now, if we assume that $\frac{\delta h}{\delta k}=\left(\begin{array}{l}h_{1} \\ h_{2}\end{array}\right)$, given that $\mathcal{H}$ is an extension, we have $H=$ $\left(\begin{array}{cc}\alpha & h_{1} \\ h_{2} & -\alpha\end{array}\right)$. Condition (3.5) results in $\left(h_{2}\right)_{x}+2 k_{1} \alpha=0$, which determines $\alpha=$ $-\frac{1}{k_{1}}\left(h_{2}\right)_{x}$ and hence $H . F$ is obtained in a similar manner. Using these values the reduced Poisson bracket is given by

$$
\{h, f\}_{R}(K)=\int_{S^{1}} B\left(F, H_{x}+[K, H]\right) d x=\int_{S^{1}}\left(\begin{array}{ll}
f_{1} & f_{2}
\end{array}\right) \mathcal{D}\left(\begin{array}{l}
h_{1} \\
h_{2}
\end{array}\right),
$$

where $D=\frac{d}{d x}$ and

$$
\mathcal{D}=\left(\begin{array}{cc}
0 & 0 \\
0 & \frac{k_{2}}{k_{1}} D+D \frac{k_{2}}{k_{1}}-\frac{1}{2} D \frac{1}{k_{1}} D \frac{1}{k_{1}} D
\end{array}\right) .
$$

If we now choose bracket (2.5) to reduce, with the choice $H_{0}=\left(\begin{array}{cc}a & b \\ c & -a\end{array}\right) \in \mathfrak{g}^{*}$, then

$$
\{h, f\}_{R}^{0}(K)=\int_{S^{1}} B\left(F,\left[H_{0}, H\right]\right) d x=\int_{S^{1}}\left(\begin{array}{ll}
f_{1} & f_{2}
\end{array}\right) \mathcal{D}_{0}\left(\begin{array}{l}
h_{1} \\
h_{2}
\end{array}\right)
$$

where

$$
\mathcal{D}_{0}=\left(\begin{array}{cc}
0 & -c D \frac{1}{k_{1}}+2 a \\
-c \frac{1}{k_{1}} D-2 a & b\left(D \frac{1}{k_{1}}+\frac{1}{k_{1}} D\right)
\end{array}\right) .
$$

The choice $a=0$ produces an invertible Poisson bracket when reduced, and so does $c=b=0$. As a result of our theorem $\{,\}_{R}^{0}$ and $\{,\}_{R}$ are compatible.

\section{Geometric evolutions with Hamiltonian invariantization}

Assume we have a geometric evolution on $\left(G \ltimes \mathbb{R}^{n}\right) / G$, that is, an evolution of curves in $\left(G \ltimes \mathbb{R}^{n}\right) / G$ which is invariant under the action of the group $G \ltimes \mathbb{R}^{n}$. It is known $([\mathrm{Q})$ that such an evolution is necessarily of the form

$$
u_{t}=R \mathbf{r}
$$

where $R$ is an invertible matrix containing in columns a classical moving frame (equivalently, a nondegenerate matrix of relative differential invariants with Jacobian weight), and where $\mathbf{r}$ is a vector of differential invariants, that is, a vector depending on the differential invariants and their derivatives with respect to $x$.

Using Theorem 1 we can write explicitly the evolution induced on the moving coframes by a geometric evolution of the form (4.1). Indeed, in our special case the action of $G$ on $\mathbb{R}^{n}$ is linear and so $g \in G$ can be identified with the Jacobian matrix $d \phi_{g}(u) \in \mathrm{GL}(n, \mathbb{R})$. Hence, if $\rho: J^{(m)}\left(\mathbb{R}^{n}\right) \rightarrow G \ltimes \mathbb{R}^{n}$ is a left moving frame and, locally, $\rho=\left(\begin{array}{cc}1 & 0 \\ \rho_{u} & \rho_{G}\end{array}\right) \in \mathrm{GL}(n+1, \mathbb{R})$ with $\rho_{G}\left(x, u^{(m)}\right) \in G$, then $\rho_{G}$ contains in columns the classical moving frame associated to the geometry.

This allows us to write the evolution induced on $\mathcal{K}$ by geometric evolutions the same way we did in M1. Let $\omega$ be the Maurer-Cartan form on $G \ltimes \mathbb{R}^{n}$.

Theorem 7. Let $\rho$ be a moving frame along $u$ and let $u$ evolve following (4.1) where $R$ is given by $\rho_{G}$. Assume that either there are no first-order differential invariants or the parameter $x$ can be chosen so that the first-order differential invariants are constant (for example $x$ would be the arc-length in the Euclidean case). Assume 
evolution (4.1) preserves the parameter $x$. Then, the component $K=\rho_{G}^{-1}\left(\rho_{G}\right)_{x}$ in the moving coframe (3.2) evolves following the equation

$$
K_{t}=T_{x}+[K, T]
$$

where $T=\rho_{G}^{-1}\left(\rho_{G}\right)_{t}$. If the parameter is fixed so that it depends on time, the preservation of $x(t)$ by evolution (4.1) is guaranteed whenever $T$ satisfies $T \Lambda=$ $\mathbf{r}_{x}+K \mathbf{r}$.

Proof. First of all, notice that, if $\rho$ is as in (3.1) with $\rho_{u}=u$, then

$$
\rho^{-1} \rho_{t}=\left(\begin{array}{cc}
0 & 0 \\
\rho_{G}^{-1}\left(\rho_{u}\right)_{t} & \rho_{G}^{-1}\left(\rho_{G}\right)_{t}
\end{array}\right)=\left(\begin{array}{cc}
0 & 0 \\
\mathbf{r} & \rho_{G}^{-1}\left(\rho_{G}\right)_{t}
\end{array}\right) .
$$

Equation (4.2) is simply the result of evaluating the structure equation

$$
d \omega+\frac{1}{2}[\omega, \omega]=0
$$

for the Maurer-Cartan form $\omega$ on the vector fields $\rho_{x}$ and $\rho_{t}$ along the curve $(u, \rho)$. Since evolution (4.1) preserves first-order differential invariants we have that, as vector fields, $\left[\rho_{x}, \rho_{t}\right]=0$ and so one obtains

$$
\frac{d}{d t}\left(\begin{array}{cc}
0 & 0 \\
\Lambda & K
\end{array}\right)=\frac{d}{d x}\left(\begin{array}{cc}
0 & 0 \\
\mathbf{r} & \rho_{G}^{-1}\left(\rho_{G}\right)_{t}
\end{array}\right)+\left[\left(\begin{array}{cc}
0 & 0 \\
\Lambda & K
\end{array}\right),\left(\begin{array}{cc}
0 & 0 \\
\mathbf{r} & \rho_{G}^{-1}\left(\rho_{G}\right)_{t}
\end{array}\right)\right],
$$

where $K=\rho_{G}^{-1}\left(\rho_{G}\right)_{x}$. If all first-order differential invariants are assumed to be constant, $\Lambda$ will be constant. If we define $T=\rho_{G}^{-1}\left(\rho_{G}\right)_{t}$, condition $0=\mathbf{r}^{\prime}+K \mathbf{r}-T \Lambda$ is the first-order invariants-preserving condition on the evolution (4.1). We conclude the proof.

An interesting aspect of this theorem is that, in all known cases, the condition $T \Lambda=\mathbf{r}_{x}+K \mathbf{r}$ and the fact that $T$ must satisfy equation (4.2) determines $T$ completely in an algebraic manner. Hence, one does not need to know $\rho$ before finding $T$.

Example 3. In the $\mathrm{SL}(2, \mathbb{R})$ case equation (4.3) becomes

$$
\left(\begin{array}{cc}
0 & 0 \\
0 & K_{t}
\end{array}\right)=\left(\begin{array}{cc}
0 & 0 \\
\mathbf{r}_{x}+K \mathbf{r}-N e_{1} & N_{x}+[K, N]
\end{array}\right) .
$$

If $\mathbf{r}=\left(r_{i}\right)$ and $T=\left(t_{i j}\right)$ we have

$$
T e_{1}=\mathbf{r}_{x}+K \mathbf{r}=\left(\begin{array}{c}
t_{11} \\
t_{21}
\end{array}\right)=\left(\begin{array}{c}
\left(r_{1}\right)_{x}+k_{2} r_{2} \\
\left(r_{2}\right)_{x}+k_{1} r_{1}
\end{array}\right)
$$

and

$$
\left(\begin{array}{cc}
0 & k_{2} \\
k_{1} & 0
\end{array}\right)=\left(\begin{array}{cc}
\left(t_{11}\right)_{x}+k_{2} t_{21}-k_{1} t_{12} & \left(t_{12}\right)_{x}-2 k_{2} t_{11} \\
\left(t_{21}\right)_{x}+2 k_{1} t_{11} & -\left(t_{11}\right)_{x}-k_{2} t_{21}+k_{1} t_{12}
\end{array}\right) .
$$

This implies $t_{12}=\frac{1}{k_{1}}\left(\left(t_{11}\right)_{x}+k_{2} t_{21}\right)$ and so $T$ is given by

$$
T=\left(\begin{array}{cc}
\left(r_{1}\right)_{x}+k_{2} r_{2} & \frac{1}{k_{1}}\left(r_{1}\right)_{x x}+\frac{1}{k_{1}}\left(k_{2} r_{2}\right)_{x}+\frac{k_{2}}{k_{1}}\left(r_{2}\right)_{x}+k_{2} r_{1} \\
\left(r_{2}\right)_{x}+k_{1} r_{1} & -\left(r_{1}\right)_{x}-k_{2} r_{2}
\end{array}\right) .
$$

Theorem 8. If there exists a Hamiltonian $h: \mathcal{K} \rightarrow \mathbb{R}$ and a local extension $\mathcal{H}$ constant on the leaves of $E$ such that

$$
\frac{\delta \mathcal{H}}{\delta M} \Lambda=\mathbf{r}_{x}+K \mathbf{r}
$$


then the invariantization of evolution (4.1) is Hamiltonian with respect to the reduced bracket $\{,\}_{R}$ and its associated Hamiltonian is $h$.

Proof. To prove this theorem we will write the evolution induced by (4.1) on $\mathcal{K}$ and compare it to the reduced Hamiltonian evolution induced on $\mathcal{K}$ by $\{,\}_{R}$ with Hamiltonian given as in the statement of the theorem. We will conclude that both vector fields are equal.

Let's denote by $\langle$,$\rangle the nondegenerate pairing of \mathcal{L} \mathfrak{g}$ with $\mathcal{L}_{\mathfrak{g}}$ ' given by the integral of the Killing form. Let $K_{t}$ be the evolution (4.2) induced on $\mathcal{K}$ by (4.1). Since $K_{t}$ is tangent to $\mathcal{K}$, if $g: \mathcal{K} \rightarrow \mathbb{R}$ is a functional, its variational derivative along $\mathcal{K}$ will be an element in the dual of the tangent to $\mathcal{K}$. Any extension constant on the leaves of $E$, call it $\mathcal{G}$, will have variational derivative with the exact same component in the direction dual to the tangent of $\mathcal{K}$. That is, as vector fields on $\mathcal{K}$,

$$
\begin{gathered}
K_{t}(g)=\left\langle K_{t}, \frac{\delta g}{\delta K}\right\rangle=\left\langle T_{x}+[K, T], \frac{\delta \mathcal{G}}{\delta M}(K)\right\rangle \\
=-\left\langle T,\left(\frac{\delta \mathcal{G}}{\delta M}(K)\right)_{x}+\left[K, \frac{\delta \mathcal{G}}{\delta M}(K)\right]\right\rangle .
\end{gathered}
$$

On the other hand, the Poisson bracket induced on $\mathcal{K}$ is given by

$$
\begin{aligned}
\{h, g\}_{R}(K) & =\xi_{h}(g)=\left\langle\left(\frac{\delta \mathcal{H}}{\delta M}(K)\right)_{x}+\left[K, \frac{\delta \mathcal{H}}{\delta M}(K)\right], \frac{\delta \mathcal{G}}{\delta M}(K)\right\rangle \\
= & -\left\langle\frac{\delta \mathcal{H}}{\delta M}(K),\left(\frac{\delta \mathcal{G}}{\delta M}(K)\right)_{x}+\left[K, \frac{\delta \mathcal{G}}{\delta M}(K)\right]\right\rangle
\end{aligned}
$$

for any extensions $\mathcal{H}$ and $\mathcal{G}$ constant on the $E$-leaves. But if $\mathcal{G}$ is constant on the leaves of $E$, the vector field $\left(\frac{\delta \mathcal{G}}{\delta M}(K)\right)_{x}+\left[K, \frac{\delta \mathcal{G}}{\delta M}(K)\right]$ is in the annihilator of $\mathfrak{n}$. If we split (as vector spaces) $\mathfrak{g}=\mathfrak{n} \oplus \mathfrak{m}$, then $\mathfrak{n}^{o}=\mathfrak{m}^{*}$. We can then conclude that, if the $\mathfrak{m}$-component of $T$ and $\frac{\delta \mathcal{H}}{\delta M}(K)$ coincide, then both vector fields $K_{t}$ and $\xi_{h}$ coincide (recall that we are identifying $\mathfrak{g}$ and $\mathfrak{g}^{*}$ using the nondegenerate Killing form).

Let $T=T_{n}+T_{m}$ and $\frac{\delta \mathcal{H}}{\delta M}=\left(\frac{\delta \mathcal{H}}{\delta M}\right)_{n}+\left(\frac{\delta \mathcal{H}}{\delta M}\right)_{m}$ be splittings given by $\mathfrak{g}=\mathfrak{n} \oplus \mathfrak{m}$. If $T_{m} \Lambda=\left(\frac{\delta \mathcal{H}}{\delta M}\right)_{m} \Lambda$, then $T_{m}-\left(\frac{\delta \mathcal{H}}{\delta M}\right)_{m} \in \mathfrak{n}$ and so $T_{m}=\left(\frac{\delta \mathcal{H}}{\delta M}\right)_{m}$. Condition (4.4) guarantees that

$$
T_{m} \Lambda=T \Lambda=\left(\frac{\delta \mathcal{H}}{\delta M}\right)_{m} \Lambda=\frac{\delta \mathcal{H}}{\delta M} \Lambda,
$$

and so we conclude the proof.

In practice, checking whether or not an evolution as in (4.1) satisfies (4.4) is not as complicated as it might seem. Since any extension will have the gradient of the original functional $h$ in the direction dual to $T \mathcal{K}$, we know what those entries are. We also know that $\left(\frac{\delta \mathcal{H}}{\delta M}\right)_{x}+\left[K, \frac{\delta \mathcal{H}}{\delta M}\right] \in \mathfrak{n}^{o}$ and, in all known examples, this condition determines $\frac{\delta \mathcal{H}}{\delta M}(K) \Lambda$ algebraically in terms of the variational derivative of $h$ and $K$. Making it equal to $\mathbf{r}_{x}+K \mathbf{r}$ usually allows you to solve for $\frac{\delta h}{\delta K}$ and to check if $h$ exists. See [M3 for examples in the Euclidean case.

Example 4. In the $\mathrm{SL}(2, \mathbb{R})$ case we know that, given $h: \mathcal{K} \rightarrow \mathbb{R}$ the variational derivative of a proper extension at $\mathcal{K}$ is given by

$$
H=\frac{\delta \mathcal{H}}{\delta M}(K)=\left(\begin{array}{cc}
-\frac{1}{2 k_{1}}\left(h_{2}\right)_{x} & h_{1} \\
h_{2} & \frac{1}{2 k_{1}}\left(h_{2}\right)_{x}
\end{array}\right) .
$$


Therefore, condition (4.4) becomes

$$
\frac{\delta \mathcal{H}}{\delta M}(K) e_{1}=\left(\begin{array}{c}
-\frac{1}{2 k_{1}}\left(h_{2}\right)_{x} \\
h_{2}
\end{array}\right)=\left(\begin{array}{c}
\left(r_{1}\right)_{x}+k_{2} r_{2} \\
\left(r_{2}\right)_{x}+k_{1} r_{1}
\end{array}\right)
$$

for some $r_{1}$ and $r_{2}$ depending on $k_{1}, k_{2}$ and their derivatives with respect to $x$. Hence, if there exists a Hamiltonian on $\mathcal{K}$ such that $h_{2}=\frac{\delta h}{\delta k_{2}}$ and $h_{2}$ satisfies (4.5), then the evolution of curves

$$
u_{t}=r_{1} u_{1}+\frac{r_{2}}{\operatorname{det}\left(u_{1}, u_{2}\right)} u_{2}
$$

will be Hamiltonian with respect to $\{,\}_{R}$.

\section{REFERENCES}

[DS] V. G. Drinfel'd and V. V. Sokolov. Lie algebras and equations of Korteweg-de Vries type, In Current problems in mathematics, Vol. 24, Itogi Nauki i Tekhniki, pages 81-180. Akad. Nauk SSSR Vsesoyuz. Inst. Nauchn. i Tekhn. Inform., Moscow, 1984. MR0760998 (86h:58071)

[F] A. Fialkow. The conformal theory of curves, Transactions of the AMS, 51, pp. 435-568, 1942. MR0006465 (3:307e)

[FO1] M. Fels, P.J. Olver. Moving coframes. I. A practical algorithm, Acta Appl. Math., pp. 99-136, 1997. MR1620769(99c:53012)

[FO2] M. Fels, P.J. Olver. Moving coframes. II. Regularization and theoretical foundations. Acta Appl. Math., pp. 127-208, 1999. MR.1681815(2000h:58024)

[Ha] R. Hasimoto. A soliton on a vortex filament, J. Fluid Mechanics, 51:477-485, 1972.

[K] S. Kobayashi. Transformation Groups in Differential Geometry, Classics in Mathematics, Springer-Verlag, New York, 1972. MR0355886 (50:8360)

[KQ1] Kai-Seng Chou and C. Qu. Integrable equations arising from motions of plane curves, Phys. D, 162(1-2): 9-33, 2002. MR1882237 (2003c:37106)

[KQ2] Kai-Seng Chou and C. Qu. Integrable equations arising from motions of plane curves II, J. Nonlinear Sci., 13(15): 487-517, 2003. MR2007575

[LP1] J. Langer and R. Perline. Poisson geometry of the filament equation, J. Nonlinear Sci., 1(1):71-93, 1991. MR.1102831 (92k:58118)

[LP2] J. Langer and R. Perline. Geometric Realizations of Fordy-Kulish Nonlinear Schrödinger Systems, Pacific Journal of Mathematics,195-1 pp. 157-177, 2000. MR1781618 (2001i:37114)

[Ma] F. Magri, A simple model of the integrable Hamiltonian equation, J. Math. Phys. 19 (1978), 1156-1162. MR0488516 (80a:35112)

[M1] G. Marí Beffa. Hamiltonian structures on the space of differential invariants of curves in flat semisimple homogeneous manifolds, submitted, 2004.

[M2] G. Marí Beffa. Poisson brackets associated to the conformal geometry of curves, To appear in the Transactions of the American Mathematical Society.

[M3] G. Marí Beffa. Poisson brackets associated to invariant evolutions of Riemannian curves, Pacific Journal of Mathematics, 215 (2004), no. 2, 357-380. MR2068787

[M4] G. Marí Beffa. The theory of differential invariants and KdV Hamiltonian evolutions, Bull. Soc. Math. France, 127(3):363-391, 1999. MR1724401 (2001m:37142)

[MSW] G. Marí Beffa, J. Sanders, J.P. Wang. Integrable Systems in Three-Dimensional Riemannian Geometry, J. Nonlinear Sc., pp. 143-167, 2002. MR1894465 (2003f:37137)

[MR] J.E. Marsden, T. Ratiu. Reduction of Poisson Manifolds, Letters in Mathematical Physics, 11: 161-169, 1986. MR0836071 (87h:58067)

[O] P.J. Olver. Equivalence, Invariance and Symmetry, Cambridge University Press, Cambridge, UK, 1995. MR1337276 (96i:58005)

[O2] P.J. Olver. Moving frames and singularities of prolonged group actions, Selecta Math, 6: 41-77 (2000). MR:1771216 (2002b:53016)

[Ov] L.V. Ovsiannikov. Group Analysis of Differential Equations. Academic Press, New York (1982). MR0668703 (83m:58082)

[PS] A. Pressley, G. Segal. Loop groups, Oxford Math. Monographs, Oxford Sc. Pub., Oxford University Press, New York, 1986. MR0900587 (88i:22049) 
[Sh] R.W. Sharpe. Differential Geometry: Cartan's Generalization of Klein's Erlangen Program, Graduate Texts in Mathematics, Springer, 1997. MR.1453120 (98m:53033)

[SW] J. Sanders and J.P. Wang. Integrable Systems in $n$-dimensional Riemannian Geometry, Moscow Mathematical Journal, 3, 2004. MR2058803(2004m:37142)

Department of Mathematics, University of Wisconsin, Madison, Wisconsin 53706

E-mail address: maribeff@math.wisc.edu 